\title{
¿POR QUÉ VILLANUEVA DE LOS INFANTES ES EL "LUGAR DE LA MANCHA" EN EL QUIJOTE?
}

\section{WHY VILLANUEVA DE LOS INFANTES IS THE "PLACE OF LA MANCHA" IN DON QUIXOTE?}

Francisco Parra Luna Universidad Complutense de Madrid. España/Spain parraluna3495@yahoo.es

Recibido/Received: 27/03/2014

Modificado/Modified: 30/05/2014

Aceptado/Accepted: 27/06/2014

\section{RESUMEN}

Se parte del hecho de que El Ingenioso Hidalgo don Quijote de la Mancha es solo una novela. Se trata sí, de una historia, de un relato ficticio, pero también en tanto que representativa de las primeras novelas realistas, describe sitios, épocas y personajes que correspondían a los siglos XVI y XVII cuando fue concebida o escrita. La Mancha, El Toboso, Barcelona, la expulsión de los moriscos por Felipe III o la mención al bandido Roque Guinart en Cataluña, demuestran que Cervantes estaba describiendo sitios, personajes y épocas reales. ¿Por qué no intentar conocer el "lugar de la Mancha" de donde salió don Quijote? En el presente trabajo, y a partir de diferentes metodologías utilizadas por el autor en trabajos anteriores, se va a pre-verificar que dicho lugar fue Villanueva de los Infantes, en la parte sureste de la provincia de Ciudad Real.

\section{PALABRAS CLAVE}

Puerto Lápice, lugar de penitencia (Sierra Morena), El Toboso, tardanzas/distancias, metodología científica.

\section{SUMARIO}

1. Introducción. 2. El problema del lenguaje. 3. Hacia el uso de metodologías científicas: el enfoque sistémico. 4. El enfoque topológico. 5. El enfoque determinista. 6. El enfoque lógico. 7. El enfoque histórico. 8. Resumen y conclusión. Bibliografía.

\begin{abstract}
This work starts from the fact that the Ingenioso Hidalgo don Quijote de La Mancha is a novel. It is a fictitious, imaginary story, but also as representative of one of the first realistic novels. It describes places, names and times which were real at the XVI century when it was written. La Mancha, El Toboso, Barcelona, the expulsion of the Moriscos by King Felipe III, or the mention of Roque Guinart in Cataluña, show that Cervantes was describing places, names and times which were real. Why, then, not to try to know which was "the place in La Mancha" where don Quixote lived? In this work, and from different methodologies used by the author in previous works, it will be pre-determined that the place in La Mancha is Villanueva de los Infantes, in the south-east part of the province of Ciudad Real in Spain.
\end{abstract}

\section{KEYWORDS}

Puerto Lápice, Place of penitence (Sierra Morena), El Toboso, lateness/distances, scientific methodology. 


\section{CONTENTS}

1. Introduction. 2. The problem of language. 3. Towards the use of scientific methodologies: The systemic approach. 4 . The topological approach. 5 The deterministic approach. 6 . The logical approach. 7. The historical approach. 8. Summary and conclusion. References.

\section{INTRODUCCIÓN}

Cuando en 2005 apareció el primer estudio científico con el título de "El Lugar de la Mancha es...." (Parra Luna et al., 2005) llevado a cabo por un equipo de la Universidad Complutense de Madrid, el mundo conocedor de El Quijote se llevó una sorpresa. No encajaba la idea de que un pueblo relativamente grande como era Villanueva de los Infantes en la época fuera el pueblo de donde salieron don Quijote y Sancho Panza y aún hoy despierta dudas en muchas personas. Se hace preciso, pues, reconsiderar el problema con la mayor claridad posible, explicitando bien los pasos metodológicos seguidos, con el fin de que quienes se acerquen al mismo con actitud científica y desinteresada, puedan comprobar o criticar tanto la metodología utilizada como el resultado obtenido. Claridad buscada que no debe estar reñida con el rigor académico aunque en el presente trabajo se persiga sobre todo la comprensión directa por cualquier lector culto interesado. Para un planteamiento más técnico véanse los trabajos incluidos en Parra Luna y Fernández Nieto (2009). De hecho y desde el punto de vista metodológico, lo más completo que puede formularse es el tratamiento sistémico que tenga en cuenta, de un lado, todas las variables que deban intervenir por significativas (distancias a El Toboso, Puerto Lápice, molinos de viento y otros sitios, subida antes de llegar al pueblo y río donde se lava la ropa viniendo desde el norte, existencia de picota, etc.); y de otro, todos los pueblos del Campo de Montiel más los que se puedan considerar comprendidos en una definición más laxa del mismo. Esto ya se hizo en dos cuadros de doble entrada: el primero cruzando 24 variables con 26 pueblos; el segundo seleccionando más cuidadosamente solo 15 variables y los 24 pueblos del Campo de Montiel (Parra Luna et al., 2005:205-265) . En ambos casos la solución fue la misma: Villanueva de los Infantes es el pueblo que cumple mayor número de requisitos. Sería la manera más global, objetiva y neutra de encarar el problema.

El método empleado debería haber sido suficiente para dejar incontestado el resultado. Pero al parecer no siempre ha sido así, por lo que el presente trabajo, aparte de volver al método Sistémico haciendo referencia a las matrices citadas, va a poner el énfasis en un proceso metodológico diferente y complementario en espera de que la solución final resulte suficientemente aclaratoria y convincente.

\section{EL PROBLEMA DEL LENGUAJE}

En principio, el quid del problema está en el lenguaje. Se tenía la idea de que el famoso "lugar de la Mancha" de cuyo nombre no quiso acordarse Cervantes, había de ser una pequeña aldea, cerca de El Toboso y con un solo cura. Y así parecía darlo a entender una lectura de la novela poco preocupada por la posibilidad de descubrirlo, e interpretación no poco justificada además ante algunas frases escritas por el propio Cervantes, como cuando Sancho dice que conoce a Aldonza Lorenzo dada la cercanía de su pueblo a El Toboso, o cuando don Quijote dice a don Álvaro Tarfe que era de una aldea próxima a donde se 
encontraban. Lenguaje casi siempre impreciso o no cuantificado a través de términos como "cerca", "próximo", "no lejos", etc., que no responde a una descripción suficientemente exacta de los desplazamientos entre los lugares reales citados en la novela. Y es que Cervantes utiliza con profusión dos tipos de lenguaje que cualquier análisis científico consagrado a esta finalidad ha de diferenciar: un lenguaje llamado "débil" por no cuantificado y no falsable (lo que comúnmente se llama subjetivo o no comprobable); y un lenguaje considerado "fuerte" por cuantificado y por lo tanto objetivo y falsable (se puede comprobar si es o no cierto).

Además, de manera querida o no querida por parte de Cervantes, existen en el texto de la novela una serie de una contradicciones entre ambos lenguajes de carácter epistemológico tener en cuenta si se persiguen resultados válidos. El caso más claro es el del Toboso: cuando después de dar a entender en varias ocasiones que viven muy cerca de este pueblo, luego resulta que tardan dos días y una noche en llegar a él y sin que medien aventuras de por medio.

Luego el análisis científico debe forzosamente comenzar por diferenciar entre estos dos tipos de lenguaje utilizados, ya que el "débil" no tiene tanto valor científico (capacidad de falseamiento) como el "fuerte". Decir, por ejemplo, que un objeto es ligero, o es pesado, carece de significación científica rigurosa, puesto que se trata de una apreciación subjetiva o proposición no falsable, la cual no se puede comprobar ni poner en duda; sin embargo, si se dice que dicho objeto pesa 8,5 kilos, la expresión resulta perfectamente falsable, o sea, se puede pesar y comprobar la validez de tal expresión. De aquí que la diferenciación de ambos tipos de lenguaje resulte previa a cualquier método sobre la localización del tan concitado "lugar" en El Quijote. Esta distinción entre los lenguajes comúnmente llamados "cualitativo" y "cuantitativo" no implica la invalidez absoluta del primero, sino su subordinación cuando contradiga al segundo. Añadiendo demás que lo cualitativo juega muchas veces un papel tan relevante como el cuantitativo si queda muchas veces repetido en el texto de la novela, como cuando se refiere al "Campo de Montiel lo que sucede hasta en cinco ocasiones.

El no respetar este fundamental principio de "falsabilidad" de las proposiciones", es lo que ha llevado a muchos estudiosos a asegurar que el "lugar de la Mancha" es cualquier pequeño pueblo que está más o menos próximo a El Toboso y sobre todo al socaire de seleccionar y conjuntar las circunstancias o factores cualitativos que "cuadraban" con algún pueblo en particular. Por este procedimiento se han conocido una veintena de estudios que aseguran que el pueblo de donde salieron don Quijote y Sancho en la novela es Quero, Puebla de Almoradiel, Miguel Esteban, Mota del Cuervo, Alcázar de San Juan, incluso Esquivias.....y un largo etcétera que llega hasta alguno ubicado lejos de El Toboso como los situados en las montañas de León, por no mencionar el caso de Argamasilla de Alba, tan tradicional como injustificadamente tenida como tal. Serie de publicaciones extraacadémicas que presentan la particularidad de anularse mutuamente sin necesidad de entrar seriamente en su crítica. Convendría por ello comenzar a exigir una apoyatura más sólida a este tipo de estudios, única manera de avanzar en la acumulación de conocimiento válido sobre el tema. Un tema por cierto no menor, como ha querido creerse, puesto que responde a un deseo explícito del propio Cervantes.

Pero ¿qué apoyatura? La validez del conocimiento depende del método empleado, y por lo tanto deben establecerse con precisión los pasos metodológicos a seguir. En este caso concreto se utilizarán cinco enfoques sucesivos que se llamaré Sistémico, Topológico, Determinista, Lógico e Histórico, no sin poner especial énfasis en cierto sitio geográfico (el 
punto en Sierra Morena donde don Quijote cumple su penitencia) que resulta clave y al cual, sorprendentemente, tampoco se le ha prestado la atención debida.

\section{HACIA EL USO DE METODOLOGÍAS CIENTÍfICAS: EL ENFOQUE SISTÉMICO}

Sobre esta carencia de metodologías ortodoxas en los estudios comentados, conviene señalar de antemano lo siguiente: "señales" sacadas de El Quijote que convengan a una u otra de las posiciones preconcebidas, seguramente se pueden encontrar bastantes. Pero la primera regla metodológica exige registrar todas las "señales" posibles, y antes de comenzar a trabajar con ellas y elaborar hipótesis alguna, preguntarse si están todas las que son y son todas las que están en el texto. Y solo a partir de un tal inventario, absolutamente completo de las "señales" o variables más relevantes, es cuando se puede elaborar un cuadro de doble entrada con todos los requisitos a satisfacer (p.e., en columnas) y todos los pueblos posibles (filas), para determinar entonces el pueblo que mayor número de requisitos satisfaga. Aún sin dar peso relativo a los requisitos (operación siempre difícil), es lo mínimo que con seriedad puede hacerse. No puede pasarse por alto ni una sola "señal" (o requisito), ni un solo pueblo que pudiera resultar candidato.

Este primer enfoque es el que se aplicó en dos ocasiones con variables y pueblos diferentes. La primera vez (Parra Luna et al, 2005), cruzando, como se ha dicho, 24 variables con 26 pueblos, y la segunda vez (Parra Luna y Fernández Nieto, 2009), cruzando una selección de 15 variables con 24 pueblos, proporcionando en ambos casos el mismo resultado. Los cuadros 1 a 42, de las páginas 205 a 265 (Parra Luna et al, 2005) dan cuenta del proceso estadístico seguido

La operación básica consistió en tratar de ver con la mayor objetividad posible cuáles de estos requisitos se adaptaban a las condiciones físicas y otras de cada uno de los 26 pueblos considerados, con lo cual resultó necesaria la visita y conocimiento geográfico de los mismos desde sus cuatro puntos cardinales.

Resultó ser el "lugar de la Mancha" aquel pueblo que acumuló mayor número de requisitos satisfechos, comprobándose que es Villanueva de los Infantes con 19 sobre 24 en primer lugar, seguido de Alcubillas y Villahermosa en segundo lugar con 15 sobre 24 . Y a pesar de que Villanueva de los Infantes solo alcanza un escaso $80 \%$ como "probabilidad" o grado de cumplimiento, al tener que ser, forzosamente, uno de estos pueblos el elegido por Cervantes, no existe otra solución posible que la recogida por dicho cruce. Cuadro general de variables/pueblos que representa una de las maneras más completas o menos criticables de determinar a qué pueblo pudo corresponder el papel de "lugar de la Mancha" en El Quijote, y operación que puede ser verificada o falsada a partir de dichos cuadros.

Este enfoque, al que llamaré "sistémico" por ser el más inclusivo, debería resultar suficientemente concluyente. No obstante, y por si todavía surgieran dudas, se siguió un proceso de métodos sucesivos que consistió en utilizar los cuatro pasos o enfoques siguientes: Topológico, Determinista, Lógico e Histórico.

\section{EL ENFOQUE TOPOLÓGICO}

Desde la perspectiva de este enfoque, tanto la ubicación del mencionado punto de penitencia de don Quijote en Sierra Morena, como la identificación del "lugar de la Mancha" que es el 
objetivo final perseguido en este trabajo, van a depender de una metodología compuesta por un axioma y cinco hipótesis de trabajo principales que será preciso explicitar desde ahora para aceptarla o rechazarla previamente a su utilización y siempre antes de conocer sus resultados. Se ruega por ello al lector la máxima posición crítica a cada uno de los siguientes postulados.

El axioma consiste en adoptar como hecho literario indiscutible la afirmación cervantina de que el pueblo de don Quijote es real y existe dado que Cervantes escribe: Este fin tuvo el Ingenioso Hidalgo de la Mancha, cuyo lugar no quiso poner Cide Hamete puntualmente, por dejar que todas las villas y lugares de la Mancha contendiesen entre sí por ahijárseles y tenérsele por suyo, ......: frase compuesta por 37 palabras que no admite, a pesar del general carácter paródico y burlesco de la novela, otras conjeturas que: a) la existencia literaria (real) de dicho pueblo según el relato; y b) el deseo de Cervantes de que se compita por localizarlo. Preciso sería volver sobre el significado literal de la frase si se pone en duda esta doble intención de Cervantes.

Las hipótesis de trabajo son las siguientes:

En primer lugar el tipo de "legua" a utilizar, pareciéndonos lógico adoptar la legua castellana de 5,5 kilómetros.

En segundo lugar, la velocidad de marcha promedio que podrían llevar las cabalgaduras de don Quijote y Sancho, que fue calculada en $31 \mathrm{kms} /$ día en comparación con los $20 \mathrm{kms}$ que proponen alguno autores y los $50 \mathrm{kms}$ que se consideraban normales en la época para una mula andando en jornada de 10 horas en verano. A la cifra de $31 \mathrm{kms} /$ día se llega a su vez por tres vías (características físicas de las cabalgaduras (Pollos, 1976); comparación con la del Caballero de la Blanca Luna en Barcelona; y estudio del sistema distancias/tiempos (Terrero, 1960).

En tercer lugar, las tardanzas en días en hacer los recorridos "pueblo de don Quijote-sitios precisados por Cervantes (Puerto Lápice, Sierra Morena, El Toboso)". Las estimaciones aproximadas de 2,42 días; 3,5 días y 2,8 días respectivamente, están justificadas en la publicación "El Enigma resuelto de El Quijote", capítulo 3 (Parra Luna y Fernández Nieto, 2009). Lógicamente, y como se mantendrá más adelante, es el momento de criticar estas aproximaciones y proponer otras que respeten igualmente la lógica y el sentido común que se desprende de las expresiones cervantinas. Si se hace con la objetividad y neutralidad requeridas posiblemente no cambien demasiado dichas estimaciones.

En cuarto lugar, don Quijote y Sancho no llegan hasta el mismo pueblo de Puerto Lápice, sino que se detienen en una choza de cabreros por llegarles la noche y cuando tenían el pueblo a la vista. ¿A qué distancia se detuvieron, y al sur del mismo puesto que procedían del Campo de Montiel? Se adoptó la cifra de $2 \mathrm{kms}$ ya que a una menor distancia no merecería la pena detenerse a pernoctar, pero bien pudieron ser tres, o cuatro o cinco kilómetros dada la llanura del terreno. Cualquier cifra podría ser válida en una horquilla razonable, por ejemplo, de 2 a 5 o $6 \mathrm{kms}$ y no por ello diferenciar demasiado el resultado final.

$\mathrm{Y}$ en quinto lugar, las dificultades orográficas de los caminos a recorrer. ¿Qué dificultades? No queda más remedio que intentar evaluar también de una forma lo más aproximada posible las dificultades de los caminos, y después de haberlos recorrido experimentalmente. Así, desde El Toboso hasta la Sierra Morena (entre El Viso y Almodóvar") el terreno es prácticamente llano en su mayoría y solo se complica al llegar a Sierra Morena. Como además la finalidad del presente trabajo es poder llegar a demostrar, compás en mano, que es posible ubicar mediante aproximaciones aceptables el "lugar de la Mancha", preciso será disminuir en unos determinados porcentajes las distancias teóricas 
(tardanza x velocidad) para convertir la distancia por caminos en "distancias en línea recta" al modo de distancias "aéreas"). Se han establecido así, y en principio, los siguientes porcentajes reductores entre 5 y $10 \%$ que parecen razonables.

a. Recorrido El Toboso-Sierra Morena $=10 \%$

b. Recorrido Almodóvar-Punto MP (mula muerta y penitencia de don Quijote) $=10 \%$

c. Recorrido "pueblo de don Quijote"-Puerto Lápice=5\%

d. Recorrido Punto MP-"pueblo de don Quijote" $=10 \%$

e. Recorrido "pueblo de don Quijote"-El Toboso $=5 \%$

¿Resultan aceptables dichos porcentajes reductivos? Es el momento de criticarlos y cambiarlos, ya que una vez que se localice el punto MP éste resultará básico, así como las cuatro hipótesis de trabajo anteriores. Solo habría que conocer medianamente las dificultades orográficas de los caminos en la época y sobre todo estar motivados desde el principio por la localización más neutral y verificable posible de este tipo de verdad literaria.

\section{UN PUNTO GEOGRÁFICO BÁSICO EN EL QUIJOTE}

En El Quijote se mencionan diversos puntos geográficos concretos como El Toboso, Puerto Lápice, Tirteafuera o Barcelona, todos los cuales tienen una localización precisa y conocida. Pero se mencionan otros que teniendo una localización no menos real, no responden a una localidad o sitio conocidos, y por lo tanto habría que determinar con precisión suficiente dichos puntos geográficos si es que afectan a la ubicación del "lugar de la Mancha". Uno de ellos, el que resulta más determinante para este fin, es el situado en Sierra Morena, en algún punto entre los meridianos que pasen por El Viso del Marqués y Almodóvar del Campo, en la provincia de Ciudad Real, pero ya situado en la provincia de Jaén. Y a este respecto Cervantes utiliza una información suficientemente "fuerte" al concretar que dicho sitio está a más de 30 leguas de El Toboso y a 8 leguas de Almodóvar del Campo. Después añadirá que se encuentra a 3,5 días del "lugar de la Mancha" (día y medio hasta la venta donde mantearon a Sancho y dos días desde dicha venta al pueblo de don Quijote). Triple información cuantificada, al parecer nunca tenida en cuenta, pero que cambia totalmente el planteamiento del problema y las perspectivas que se venían teniendo sobre el pueblo de don Quijote y Sancho.

$\mathrm{Y}$ es mediante la aceptación o modificación de las cinco hipótesis descritas, como es posible comenzar por ubicar ese punto geográfico situado en Sierra Morena que encierra la clave para poder determinar el "lugar de la Mancha". Me refiero al sitio donde don Quijote y Sancho se encuentran con el cabrero en el episodio de la mula muerta y el personaje de Cardenio, así como poco después el de la penitencia de don Quijote y la despedida de Sancho hacia El Toboso.

Se debe proceder, pues, a determinar individualmente los puntos "M" (mula muerta) y "P" (penitencia de don Quijote). Se sabe que el primero está a 8 leguas de Almodóvar, y el segundo a más de 30 leguas de El Toboso, pero entre ambos sitios no debe existir mucha distancia puesto que ésta equivaldría solo al tiempo que se tarda en pronunciar las cuatro páginas de conversación (y quizás algo más) que median entre la frase: Señor don Quijote, vuestra merced me eche su bendición..... y la frase Llegaron en estas pláticas al pie de una alta montaña...... en el capítulo XXV de la primera parte de El Quijote. Por otra parte, no se sabe hacia dónde se desplazaron desde el punto "M" de la mula muerta (norte, sur, este, oeste), y por consiguiente el mínimo error para determinar el punto "P" (penitencia) se comete cuando se integran y funden ambos sitios en uno nuevo que se llame "MP". Se debe 
determinar, pues, ese punto geográfico que esté a más de 30 leguas de El Toboso y a 8 de Almodóvar del Campo como nuevo "sitio" fundamental que complete los de Puerto Lápice y El Toboso.

Pero "más de 30 leguas”, ¿cuanto más? Serían suficientes 31 leguas, pero dado que aplicando las hipótesis anteriores proporciona una distancias de $31 \times 5,5 \mathrm{kms}=170,5$ la cual se debe reducir en su 10\%, la distancia en línea recta sería de $153,5 \mathrm{kms}$. Y teniendo en cuenta que dicho punto debe estar situado en Andalucía (según frases del cabrero y Cardenio), se puede comprobar que la distancia mínima a aplicar sería del orden de las 32 leguas, lo que permite situar el punto más claramente ya en la provincia de Jaén (en "esta Andalucía" como se repite hasta en tres ocasiones en el texto de la novela). Luego el punto básico "P" (un punto que integraría los llamados "M" y "P" dadas las consideraciones anteriores, sería el proporcionado por el cruce entre:

a. 32 leguas desde El Toboso x 5,5 kms=176 kms que disminuidos en su $10 \%$ da 158,4 (aprox. $158 \mathrm{kms}$ ), y

b. 8 leguas desde Almodóvar del Campo x 5,5 kms= $44 \mathrm{kms}$ que disminuidos en su $10 \%$ da $39,6 \mathrm{kms}$ (aprox. $40 \mathrm{kms}$ )

Trácense ahora mediante compás sendos círculos con radios de 158 y 39,6 kms desde las poblaciones citadas $\mathrm{y}$ el punto geográfico resultante, siempre aproximado, pero absolutamente básico para determinar el "lugar de la Mancha", estaría situado en la cabecera del pantano del Jándula, al sur de Solana del Pino y ya en la provincia de Jaén. Este es el punto geográfico, kilómetro arriba o abajo, izquierda o derecha, que resulta tan ignorado como fundamental para llegar a conocer el "lugar de la Mancha"

\section{LA UBICACIÓN DEL "LUGAR DE LA MANCHA"}

Disponemos así de tres puntos geográficos concretos: Puerto Lápice, cabecera del pantano del Jándula y El Toboso, dados por Cervantes con suficiente precisión, y además se conocen las distancias (tardanzas en días $\mathrm{x}$ velocidad de las cabalgaduras) que median entre los tres puntos y el supuesto "lugar de la Mancha", es decir:

2,42 días desde el pueblo de don Quijote hasta Puerto Lápice si se acepta la aproximación final adoptada (Parra Luna y Fernández Nieto, 2009:95-115)

3,50 días desde el punto MP hasta el pueblo de don Quijote la cual se descompone en:

1,5 días desde el punto MP hasta la venta donde mantean a Sancho Panza, (tres días has tardado en ir y venir desde aquí al Toboso, habiendo más de treinta leguas según expresión de don Quijote en el capítulo XXXI de la primera parte) la cual no puede ser otra que la histórica venta Yruela (muy activa en la época por estar en camino de paso hacia Andalucía, situada en la parte norte del paso del Muradal en Ciudad Real y muy próxima a la actual Venta de Cárdenas).

2 días desde dicha Venta hasta el pueblo de don Quijote según expresión del cura (no está a más de dos jornadas de aquí) en el capítulo XXXVII de la primera parte cuando planeaban regresar al mismo para dejar a don Quijote.

2,80 días desde el pueblo de don Quijote hasta El Toboso (si se acepta la aproximación final adoptada (Parra Luna y Fernández Nieto, 2009:105).

Con esta información, derivada de las tardanzas entre sitios concretos definidas con suficiente exactitud por Cervantes, es el momento de emplear el acercamiento que he llamado Topológico, pero precisando en este trabajo las diferencias entre las distancias 
teóricas y las ajustadas (en línea recta) para utilización del compás. Los porcentajes de reducción empleados en el presente trabajo juegan así un papel fundamental.

Método que ha consistido en considerar, lo más rigurosamente posible, la geografía por donde caminaron don Quijote y Sancho Panza en relación a determinar el pueblo de donde partieron según las descripciones (variablemente explícitas) de Cervantes.

Comparando así las distancias teóricas (tardanza x velocidad) con las previstas en línea recta tenemos el cuadro 1 sobre distancias hacia y desde el todavía desconocido pueblo de don Quijote:

Cuadro 1: Distancias para compás sobre bases teóricas hacia y desde el pueblo de don Quijote

\begin{tabular}{|l|l|}
\hline Distancia teórica & Distancia para compás (según \% reductores) \\
\hline Puerto Lápice & \\
\hline 2,42 días x $31 \mathrm{kms}=75 \mathrm{kms}$ & $75-5 \%=71,3 \mathrm{kms}$ \\
\hline Desde Sierra Morena $($ PUNTO P) & \\
\hline 3,5 días x $31 \mathrm{kms}=108,5 \mathrm{kms}$ & $108,5-10 \%=97,7 \mathrm{kms}$ \\
\hline Hasta El Toboso & \\
\hline 2,8 días $\times 31 \mathrm{kms}=86,8 \mathrm{kms}$ & $86,8-5 \%=82,8 \mathrm{kms}$ \\
\hline
\end{tabular}

Fuente: Elaboración propia

Trácense mediante compás los tres círculos con sus correspondientes radios de 71,3; 97,7; y 82,8 kms, desde los tres sitios geográficos indicados (Puerto Lápice, Punto P en Sierra Morena y El Toboso), y véase en qué punto geográfico confluyen, por ejemplo en mapa de Michelín 2004, escala $1 \mathrm{~mm} .=1 \mathrm{~km}$. Se podrá apreciar gráficamente cómo queda un pequeño triángulo al norte de Villanueva de los Infantes (Ciudad Real), capital política del histórico Campo de Montiel y su centro geográfico aproximado, localización que viene a coincidir con anteriores estudios, muchos de ellos de carácter matemático (Girón y Ríos, 2006), que sitúan exactamente el "lugar de la Mancha" en la llamada "Casa Mariquita", a unos dos kilómetros al norte de Villanueva de los Infantes junto a la carretera hacia La Solana.

Puede dar la impresión de que estas medidas están cuadradas previamente para que proporcione este resultado, pero no es así. Repásense y modifíquense las cinco hipótesis de trabajo utilizadas y se comprobará que cualquier estimación, si efectuada con sentido común y estricta motivación científica, proporcionará siempre un punto que caerá, dentro, o en un alrededor muy próximo, del pequeño triángulo que indefectiblemente se situará en el centro geográfico del Campo de Montiel entre los pueblos de Alcubillas, Villanueva de los Infantes, Fuenllana y Carrizosa.

Resultado que permite una primera comprobación utilizando las distancias según las actuales carreteras y caminos como señala ahora el cuadro 2 .

Cuadro 2: Comparación de distancias por carretera y para uso del compás.

\begin{tabular}{|l|l|l|}
\hline & Distancia por carreteras $/$ caminos & distancias para compás \\
\hline Desde Puerto Lápice & $32+12+29=73 \mathrm{kms}$ & $73-5 \%=69,4 \mathrm{kms}$ \\
\hline Desde Sierra Morena & $45+11+20+35=111 \mathrm{kms}$ & $111-10 \%=99,9 \mathrm{kms}$ \\
\hline Hasta El Toboso & $12+30+45=87 \mathrm{kms}$ & $87-5 \%=82,7 \mathrm{kms}$ \\
\hline
\end{tabular}

Fuente: elaboración propia

Comprobándose así que las distancias reales por carreteras/caminos según el mapa Michelín citado, reducidas en sus correspondientes porcentajes para uso del compás, proporcionan idéntico resultado: el área central del Campo de Montiel. 
Así, pues, este área será la resultante con cualquier aproximación o hipótesis utilizada, por lo que el segundo de los cinco enfoques metodológicos previstos, el Topológico, está dado, y en consecuencia, lo único que se puede asegurar hasta el momento con un nivel de seguridad aceptable es que el "lugar de la Mancha" en el Quijote se encuentra dentro del triángulo formado por esos cuatro pueblos mencionados en el centro del Campo de Montiel, y dado que se trata de localizar un pueblo y no un cortijo u otro tipo de agrupación poblacional, forzosamente ha de ser alguno de los cuatro pueblos que delimitan dicha área. De hecho, y dado que el pueblo más próximo a la "Casa Mariquita" es Villanueva de los Infantes (solo a dos kms), por el momento al menos, sería prueba más que suficiente para identificar este último pueblo como la patria de don Quijote. No obstante, se va a seguir considerando como espacio geográfico "cuna de don Quijote" solo el área de los cuatro pueblos mencionados para mayor seguridad.

\section{EL ENFOQUE DETERMINISTA}

Una vez limitados los pueblos topológicamente posibles a los cuatro mencionados (Alcubillas, Villanueva de los Infantes, Fuenllana y Carrizosa), el próximo paso consiste en determinar cuáles de ellos se encuentran en una dirección forzosa hacia Cartagena partiendo de la parte norte de Sierra Morena (venta Yruela cerca de Venta de Cárdenas) ya en la provincia de Ciudad Real.

Sucedió en la novela que estando el cura y demás acompañantes situados en la venta donde mantean a Sancho Panza (en cualquier caso en algún sitio al norte de Sierra Morena (a no mayor distancia de 1,5 díasx31 kms=46,5 kms), en camino de paso hacia Andalucía y en meridiano próximo al de El Viso del Marqués en dirección lógica hacia El Toboso), y cuando deben dirigirse a Cartagena para embarcar a don Quijote hacia el Reino Micomicón, el cura pronuncia la siguiente frase, absolutamente determinante: si es así por la mitad de mi pueblo hemos de pasar (cap. XXIX de la primera parte). Lógicamente el cura ha de referirse al pueblo de don Quijote (puesto que se trataba de dejar al caballero en su casa).Y desde esa posición geográfica y teniendo en cuenta la estructura de caminos existente, la vía lógica a tomar sería la llamada "Vía de la Plata" Este-Oeste (Rodríguez Castillo, 1999) que viniendo de Almadén enlazaba con Valdepeñas, Villanueva de los Infantes, Alcaraz, Hellín,...hasta Cartagena. Camino que por cierto debió recorrer Cervantes cuando en 1581 se dirigió desde Tomar (Portugal) a Oran con una misión diplomática de Felipe II.

Siendo esta la situación geográfica, se dirigirían desde la venta Yruela (en el lado oeste de Ventas de Cárdenas) hacia el norte (presumiblemente vía Almuradiel, Castellar de Santiago, Alcubillas o Cózar, Villanueva de los Infantes, Fuenllana,...Alcaraz,...), para alcanzar la vía principal Ciudad Real-Murcia lo antes posible, al tiempo que minimizasen la distancia hasta el pueblo de don Quijote, por lo que en cualquiera de los casos y viendo la situación de esta vía, el pueblo de Carrizosa quedaría descartado como "lugar de la Mancha", ya que resultaría absurdo subir hacia el norte (unos $10 \mathrm{kms}$ al norte del tramo Valdepeñas-Alcaraz) para regresar de nuevo hacia el sur. Desde esta perspectiva, pues, quedarían con posibilidades todavía de ser el "lugar de la mancha" Alcubillas, Villanueva de los Infantes y Fuenllana. 


\section{EL ENFOQUE LÓGICO}

Consiste ahora en considerar cual de los tres pueblos restantes cumplía ciertas características que deberían resultar decisivas según el texto de la novela. Todo induce a pensar a partir de las mismas que el pueblo de don Quijote debía ser un pueblo lo suficientemente grande como para admitir los siguientes hechos descritos en El Quijote:

a. La parada de una compañía de soldados (según carta de Teresa Panza a Sancho en el cap. LII de la segunda parte) se supone que para pernoctar y aprovisionarse, lo que no era lógico que hiciesen ni en Alcubillas ni en Fuenllana dada la proximidad de ambos a Villanueva de los Infantes mejor dotada para estas necesidades.

b. Se produce además el hecho de que "con los soldados se van tres mozas del pueblo", lo que da idea de que debe tratarse de un pueblo lo suficientemente poblado y socialmente complejo para darse esta circunstancia.

c. La portentosa cultura literaria demostrada por el Cura con motivo de la revisión de la biblioteca de don Quijote, así como por la sorprendente del barbero (que incluso tiene uno de los libros descubiertos en versión italiana), hace pensar en un ambiente cultural más propio de pueblo grande que de pequeña aldea.

Todo lo cual determina que el pueblo no pueda ser otro que el Villanueva de los Infantes de la época, y cuya complejidad social se derivaba de las circunstancias históricas que se mencionarán en el punto siguiente.

Pero ya desde la lógica de este tercer enfoque, la determinación de Villanueva de los Infantes como el "lugar de la Mancha" en El Quijote quedaría prácticamente cerrada. Pueblo ciertamente inesperado según la literatura existente sobre El Quijote a lo largo de los cuatro siglos pasados, pero que se presenta a estas alturas del método como escasamente discutible.

\section{EL ENFOQUE HISTÓRICO}

Por si los acercamientos sistémico, topológico, determinista y lógico no resultaran suficientes, se dieron en la Villanueva de los Infantes de la época unas circunstancias históricas bastante explicativas. Comenzando por los cinco conventos existentes (el de los Trinitarios en construcción), los casi 200 clérigos viviendo en el pueblo, quizás otros tantos hidalgos o hijosdalgo como lo demuestran los 190 escudos nobiliarios existentes, así como la serie de oficiales, políticos y escribanos que necesitaba el papel político que jugaba este pueblo a finales del siglo XVI como cabecera judicial del partido. Estructura religiosocaballeresca y social ciertamente compleja, problemática y apropiada, para que de allí surgiera un personaje un tanto desequilibrado como don Quijote.

A lo que hay que añadir tres hechos históricos que muy bien pudieron inspirar a Cervantes (cuando pasó por el centro del Campo de Montiel como sugiere su viaje Tomar-Cartagena y la placa encontrada como asistente a las Bodas de Camacho), la personalidad de don Quijote en base a la de tres naturales de Villanueva de los Infantes: Santo Tomas de Villanueva, Fray Tomás de la Virgen, y el tenido por loco Juan de León, quienes como se ha dicho en trabajos anteriores (Parra Luna y Fernández Nieto, 2009) eran una especie de "santos-locos", de caridad y sapiencia los dos primeros y de justicia el segundo. Una santidad tan fuerte, prestigiosa y conocida como la que ejercieron Santo Tomas de Villanueva y Fray Tomás de la Virgen; y una personalidad tan anormal, pre-quijotesca, y sobre todo tan escandalosamente juzgada y ejecutada en Villanueva de los Infantes como la de Juan León, con posterior intervención del emperador Carlos I, condenando y desterrando al entonces alcalde de 
Villanueva de los Infantes, Doctor Juan de la Cueva, forzosamente debió provocar un fuerte impacto en la zona perdurable durante décadas y ser motivo de conocimiento por parte de Cervantes cuando muy presumiblemente pasó por allí.

Quedando así aún más cerrado el círculo que nos llevaría a asegurar con un mínimo margen de error que Villanueva de los Infantes, centro geográfico y capital histórica del Campo de Montiel, fue el "lugar de la Mancha" en la novela del Ingenioso Hidalgo.

Desde la perspectiva histórica, ningún otro pueblo, no solo entre los cuatro determinados por el enfoque topológico, sino del Campo de Montiel, de la Mancha e incluso de toda España, reunió a un tiempo estas tres circunstancias históricas (estructura social compleja, Santo Tomás, Fray Tomás de la Virgen y Juan de León) que se produjeron en Villanueva de los Infantes y justamente unos años antes (o durante) de que Cervantes escribiera su novela.

\section{RESUMEN Y CONCLUSIÓN}

Resulta posible por lo expuesto responder con suficiente precisión a la pregunta del título. En primer lugar, porque El Quijote, desde la perspectiva específica de identificar el "lugar de la Mancha", forma un sistema de proposiciones que bajo un tratamiento sistémico riguroso ofrecen una solución verificable. Bastaría incluso con comparar los escasos factores que no cuadran con Villanueva de los Infantes (cercanía a El Toboso o a Quintanar, molinos de viento, arroyo-lavadero y algún otro), con los que sí cuadran (sistema de distancias-tiempos y serie de características culturales y otras de este pueblo) para dejar una primera solución bien apuntada. Las dos matrices de "factores/pueblos" referenciadas agrupando todos los factores posibles, favorables y desfavorables para los pueblos comprendidos, lo demuestran.

Pero en este trabajo se ha añadido otra vía complementaria, descompuesta en los enfoques topológico, determinista, lógico e histórico, que además de resultar más explicativa, va encaminando deductivamente el resultado hacia: primero, el Campo de Montiel "como debe ser", según la justa expresión de Rodríguez Castillo (1999) y debido a las cinco menciones explícitas de Cervantes en el texto; segundo, hacia un área central reducida del mismo delimitada por Alcubillas, Villanueva de los Infantes, Fuenllana y Carrizosa, debido a las tardanzas entre puntos geográficos precisos dados por Cervantes; tercero, eliminando la posibilidad de Carrizosa por su situación al norte de la vía Valdepeñas-Alcaraz; y cuarto, concretándolo en Villanueva de los Infantes, centro geográfico, histórico y político del Campo de Montiel, a causa de la serie de circunstancias descritas en El Quijote que solo confluyen en dicho pueblo.

Por todo ello, y a partir del conjunto de métodos empleados, se puede enunciar como teoría científica válida, o si prefiere como hipótesis perfectamente falsable, que Villanueva de los Infantes, en el centro del Campo de Montiel, provincia de Ciudad Real, es el tan concitado "lugar de la Mancha" en El Quijote.

Ya solo queda esperar que con el tiempo (que todo lo aclara como decía don Quijote), aparezcan otras publicaciones que, con las garantías académicas y científicas necesarias, demuestren que este pueblo no fue, si no otro, el "lugar de la Mancha". El día que eso se produzca todos estaremos de enhorabuena, y el primero el autor de este artículo, siquiera sea por haber contribuido modestamente a profundizar en el tema. El progreso científico consiste en probar la falsedad de una teoría para sustituirla por otra que suponga un superior conocimiento en aras de un mayor enriquecimiento y utilidad para la sociedad. Por ello, en la 
bibliografía que sigue a continuación se añaden trabajos no citados en el texto pero que pueden resultar de interés para el lector a la hora de formular su crítica.

\section{BIBLIOGRAFÍA}

CABALlERO, F. (1918). Pericia geográfica de Miguel de Cervantes. Madrid: Imprenta de Artillería. CASELLES, A. et al. (2009). "The Kinematics of don Quixote and the identity of the Place in La Mancha: A systemic Approach", en Revista Internacional de Sistemas, 16: 7-16.

EISENBERG, D. (2003). Vida de Cervantes. Alicante: Biblioteca virtual Miguel de Cervantes.

FERNÁNDEZ NIETO, M. (2009). "El lugar de la Mancha y los mapas e itinerarios trazados de la Ruta de don Quijote”. En F. Parra Luna y M. Fernández Nieto. El Enigma Resuelto del Quijote: un debate sobre el Lugar de la Mancha. Alcalá: Universidad de Alcalá.

GIMÉNEZ SERRANO, J. (1948). Un paseo a la patria de don Quijote. Madrid: Seminario Pintoresco. GIRÓN, F. J. y RÍOS, M. J. (2006). "La determinación del lugar de la Mancha como problema estadístico", en Boletín de la Sociedad de Estadística e Investigación Operativa, 22, 1: 23-29.

LIGERO MÓSTOLES, A. (1991). La Mancha de don Quijote. Alcázar de San Juan: Ayuntamiento.

MARTÍNEZ DE LA ROSA, F. (2009). "La determinación del lugar de la Mancha en el Quijote: un modelo matemático sobre nuevas hipótesis de tardanzas". En F. Parra Luna y M. Fernández Nieto. El Enigma Resuelto del Quijote: un debate sobre el Lugar de la Mancha. Alcalá: Universidad de Alcalá. POLLOS, L. (1976). Las cabalgaduras de don Quijote y Sancho. Madrid: UCM-Facultad de Veterinaria. MORÓN ARROYO, C. (2009). "Cuyo lugar no quiso poner Cide Hamete". En F. Parra Luna y M. Fernández Nieto. El Enigma Resuelto del Quijote: un debate sobre el Lugar de la Mancha. Alcalá: Universidad de Alcalá.

MUÑOZ ROMERO, J. (2001). La única y verdadera ruta de don Quijote. Toledo: Ledoria.

NOVO, P. (1944). "Andanzas de don Quijote en tierra manchega”, en Boletín de la Sociedad Manchega de Excursiones, tomo LII, 1944.

PANADERO MOYA, M. (1997). Don Quijote en América. (s.1.): Universidad de Castilla La Mancha.

PARRA LUNA, F. et al. (2005). El lugar de la Mancha es....el Quijote como un sistema de Distancias/tiempos. Madrid: Universidad Complutense.

PARRA LUNA, F. y FERNÁNDEZ NIETO, M. (2009). El enigma resuelto del Quijote. Alcalá de Henares: Universidad de Alcalá.

RODRÍGUEZ CASTILlO, J. (1999). Don Quijote por el Campo de Montiel (como debe ser).Villanueva de los Infantes: Asociación de Amigos del Campo de Montiel.

ROMÁN ALHAMBRA, L. M. (2012). Mi vecino Alonso. Alcázar de San Juan: Ayuntamiento.

SÁNCHEZ SÁNCHEZ, J. (2009). "La imprecisión geográfica del Quijote y la búsqueda del lugar de la Mancha”. En F. Parra Luna y M. Fernández Nieto. El Enigma Resuelto del Quijote: un debate sobre el Lugar de la Mancha. Alcalá: Universidad de Alcalá.

SERRANO VICENS, R. (1972). Ruta y patria de don Quijote. Madrid: Diputación de Cuenca.

TERRERO, J. (1959). La ruta de don Quijote. Madrid: Imprenta Viuda de Galo Sáez.

TERRERO, J. (1960). "Las rutas de las tres salidas de don Quijote de la Mancha", en Anales Cervantinos, VIII, 1959-60: 1-40.

TORRES YAGÜES, F. (1962). La ruta de don Quijote. Madrid: Sociedad Cervantina, Gráficas Yagües. 


\section{Breve currículo:}

\section{Francisco Parra Luna}

Catedrático Emérito de la Universidad Complutense, Académico de Número de la "International Academy for Systems and Cybernetic Sciences", Excoordinador del Theme "Cybernetics and Systems" en la "International Enciclopedia of Life Support Systems" de la UNESCO; Fundador del "Working Group on Systems Theory and Sociocybernetics" (actual RC51) de la Asociación Internacional de Sociología. Autor de 23 libros y numerosos artículos. Acredita diversos premios y distinciones. 